\title{
The Frobenius-Schur indicator in Conformal Field Theory
}

\author{
Peter Bantay ${ }^{1}, 2$ \\ Mathematics Department, University of California at Santa Cruz
}

\begin{abstract}
An analogue of the classical Frobenius-Schur indicator is introduced in order to distinguish between real and pseudo-real self-conjugate primary fields, and an explicit expression for this quantity is derived from the trace of the braiding operator.
\end{abstract}

\footnotetext{
1 On leave from the Institute for Theoretical Physics, Eötvös University

,2 Partially supported by OTKA T016251
} 
The distinction between real and pseudo-real primary fields in a CFT had been known for a long time [1]. It corresponds to the different behavior of the holomorphic blocks of the two-point functions under braiding, and amounts to a difference in sign. A similar pattern exists for the self-conjugate superselection sectors of two-dimensional QFT-s and the representations of the related Rational Hopf-algebras [2]. The aim of this paper is to show that the above distinction is already encoded in the standard data of the CFT under study, i.e. the fusion rules, the quantum dimensions and the conformal weights of the primary fields.

An analogous classification exists in group representation theory, where one distinguishes between real, complex and quaternionic representations ( the latter are sometimes called pseudo-real ) according to the realizability of the given representation with matrices whose elements lie in the respective (skew)-field [3]. It is usual to introduce the so-called Frobenius-Schur indicator for irreducible representations, which is +1 for real, 0 for complex, and -1 for quaternionic representations, and there is a simple formula that allows to compute it from the character of the representation. In fact, the Frobenius-Schur indicator is not directly related to the reality properties of the representations; it is telling us whether there is an invariant bilinear form, and if there is one, whether it is symmetric or anti-symmetric. By a well-known theorem this may be translated into an assertion about the reality properties.

We can try to mimic the above situation by introducing for the primary fields of a CFT the Frobenius-Schur indicator $\nu_{p}$, which is +1 if the field $p$ is real, -1 if it is pseudo-real, and 0 if it is complex, i.e. different from its charge conjugate $\bar{p}$. 
What is truly remarkable about the Frobenius-Schur indicator is that there is an explicit formula expressing it in terms of such standard quantities as the fusion rules, the (quantum) dimensions and the conformal weights of the primary fields. The explicit expression for the indicator reads

$$
\nu_{p}=\sum_{r, s} N_{r s}^{p} S_{0 r} S_{0 s} \frac{\omega_{r}^{2}}{\omega_{s}^{2}}
$$

where $\omega_{p}=\exp \left(2 \pi \imath \Delta_{p}\right)$ is the exponentiated conformal weight of the primary field $p$.

To understand the origin of the above formula, let's consider the braiding of $p$ with itself. Suppose that the primary field $q$ appears in the OPE of $p$ with itself, i.e. $N_{p p}^{q}>0$, and let's consider the operator $\mathcal{R}_{p p}^{[q]}$ that braids the two copies of $p$ in the intermediate channel $q$. The braiding has as its square the monodromy which we know to be diagonal with eigenvalue $\omega_{q} \omega_{p}^{-2}$, i.e.

$$
\mathcal{R}_{p p}^{[q] 2}=\frac{\omega_{q}}{\omega_{p}^{2}} \mathbf{1}
$$

from which it follows that the possible eigenvalues of $\mathcal{R}_{p p}^{[q]}$ are $\pm \omega_{q}^{1 / 2} \omega_{p}^{-1}$, and should we know the trace of $\mathcal{R}_{p p}^{[q]}$ we would know the multiplicities of these eigenvalues as well. But the trace may be computed, and the result is

$$
\operatorname{Tr}\left(\mathcal{R}_{p p}^{[q]}\right)=\omega_{p}^{-1} \sum_{r, s} N_{r s}^{p} S_{q r} S_{0 s} \frac{\omega_{s}^{2}}{\omega_{r}^{2}}
$$

We are now ready to express the multiplicity of the eigenvalue $\pm \omega_{q}^{1 / 2} \omega_{p}^{-1}$ of $\mathcal{R}_{p p}^{[q]}$ as

$$
\frac{1}{2}\left(N_{p p}^{q} \pm \omega_{q}^{-1 / 2} \sum_{r, s} N_{r s}^{p} S_{q r} S_{0 s} \frac{\omega_{s}^{2}}{\omega_{r}^{2}}\right)
$$


Note that by the above reasoning the value of the expression

$$
\mathcal{Z}(p, q)=\omega_{q}^{-1 / 2} \sum_{r, s} N_{r s}^{p} S_{q r} S_{0 s} \frac{\omega_{s}^{2}}{\omega_{r}^{2}}
$$

should be an integer of the same parity as $N_{p p}^{q}$ and whose absolute value is not greater than $N_{p p}^{q}$. We remark that these integrality conditions could prove very effective in the classification program, because they rule out many possibilities for prospective fusion rule algebras that pass all other tests.

Returning to the Frobenius-Schur indicator, we have to consider the special case $q=0$ in the above reasoning, because we are interested in the behavior of the two-point function. In this case $N_{p p}^{0}=\delta_{p, \bar{p}}$, and it follows from the remarks following (5) that $\mathcal{Z}(p, 0)=0$ if $p \neq \bar{p}$, and $\mathcal{Z}(p, 0)= \pm 1$ if $p=\bar{p}$ depending on the eigenvalue of the braiding, i.e. the symmetry ( or anti-symmetry) of the two-point block. But this is exactly the three-fold classification that led us to define the indicator, proving our assertion that $\nu_{p}=\mathcal{Z}(p, 0)$.

The Frobenius-Schur indicator is especially simple to compute for simple currents, i.e. primary fields whose (quantum) dimension is 1. It turns out that in this case

$$
\nu_{\epsilon}=\omega_{\epsilon}^{2} \delta_{\epsilon, \bar{\epsilon}}
$$

That is, the conformal weight of a real simple current is an integer or halfinteger, while that of a pseudo-real one is $\pm \frac{1}{4}$ modulo integers. Actually, Eq.(6) is but a special case of the more general relation

$$
\nu_{\epsilon p}=\omega_{\epsilon}^{2} \nu_{p}
$$

valid for any self-conjugate simple current $\epsilon$. 


\section{References :}

[1] Alvarez-Gaume, Gomez and Sierra : Nucl. Phys. B319, (1989), 155.

Moore and Seiberg : Phys. Lett B212 (1988), 451.

[2] Fredenhagen, Rehren and Schroer : Rev. Math. Phys. 113, spec. issue (1992).

Fuchs, Ganchev and Vecsernyes : International Journal of Modern Physics A10, (1995), 3431.

[3] Isaacs, I. M. : Character Theory of Finite Groups, Academic Press, 1976.

Kirillov, A. A. : Elements of the Theory of Representations, Springer, 1976. 